\title{
Ssciendo
}

Int. J. Appl. Math. Comput. Sci., 2019, Vol. 29, No. 2, 305-310

DOI: $10.2478 /$ amcs-2019-0022

\section{POSITIVITY OF FRACTIONAL DESCRIPTOR LINEAR DISCRETE-TIME SYSTEMS}

\author{
TADEUSZ KACZOREK ${ }^{a}$ \\ ${ }^{a}$ Faculty of Electrical Engineering \\ Białystok University of Technology, Wiejska 45D, 15-351 Białystok, Poland \\ e-mail: kaczorek@ee.pw.edu.pl
}

\begin{abstract}
The positivity of fractional descriptor linear discrete-time systems is investigated. The solution to the state equation of the systems is derived. Necessary and sufficient conditions for the positivity of fractional descriptor linear discrete-time systems are established. The discussion is illustrated with numerical examples.
\end{abstract}

Keywords: fractional, descriptor, linear, discrete-time, system, stability, solution, positivity.

\section{Introduction}

A dynamical system is called positive if its state variables take nonnegative values for all nonnegative inputs and nonnegative initial conditions. Positive linear systems were investigated by Berman and Plemmons (1994), Farina and Rinaldi (2000) or Kaczorek (2002), who also studied positive nonlinear systems (Kaczorek, 2016; 2015a; 2014; 2015b; 2015c).

Examples of positive systems are industrial processes involving chemical reactors, heat exchangers and distillation columns, storage systems, compartmental systems, water and atmospheric pollution models. A variety of models having positive linear behavior can be found in engineering, management science, economics, social sciences, biology and medicine, etc.

Linear systems with different fractional orders were addressed by Busłowicz (2012), Kaczorek (2010; 2011a) and Sajewski (2016). Descriptor (singular) linear systems were analyzed by Borawski (2018), Kaczorek (2014; 2016b; 2019; 2012; 1997; 1993) or Ali and Diego (2012), and the stability of a class of nonlinear fractional-order systems was studied by Kaczorek (2016a; 2011b) or Xiang-Jun et al. (2008). Fractional positive continuous-time linear systems and their reachability were addressed by Kaczorek (2008). Application of the Drazin inverse to the analysis of descriptor fractional discrete-time linear systems was presented by Kaczorek (2013), while the stability of discrete-time switched systems with unstable subsystems was studied by Zhang et al. (2014a). Robust stabilization of discrete-time positive switched systems with uncertainties was addressed by Zhang et al. (2014b). A comparison of three methods of analysis of descriptor fractional systems was presented by Sajewski (2016a). The stability of linear fractional order systems with delays was analyzed by Busłowicz (2008), and simple conditions for practical stability of positive fractional systems were proposed by Busłowicz and Kaczorek (2009). The stability of interval positive continuous-time linear systems was addressed by Kaczorek (2018). Positive controllability of positive dynamical systems was considered by Klamka (2002), while some remarks on stability of positive linear systems were given by Mitkowski (2000), along with dynamical properties of Metzler systems (Mitkowski, 2008).

In this paper the positivity of fractional descriptor discrete-time linear systems will be investigated. The paper is organized as follows. In Section 2 basic definitions of the Drazin inverse of matrices are recalled and the solution to the system state equation is derived. Necessary and sufficient conditions for the positivity of fractional descriptor linear discrete-time systems are established in Section 3. Concluding remarks are given in Section 4.

The following notation will be used: $\mathbb{R}$, the set of real numbers; $\mathbb{R}^{n \times m}$, the set of $n \times m$ real matrices; $\mathbb{R}_{+}^{n \times m}$, the set of $n \times m$ real matrices with nonnegative entries and $\mathbb{R}_{+}^{n}=\mathbb{R}_{+}^{n \times 1} ; I_{n}$, the $n \times n$ identity matrix. 


\section{Fractional descriptor linear discrete-time systems}

Consider the fractional descriptor linear system

$$
\begin{aligned}
E \Delta^{\alpha} x_{i+1}= & A x_{i}+B u_{i}, \\
& i \in \mathbb{Z}_{+}=0,1,2, \ldots, \quad 0<\alpha<1, \\
y_{i}= & C x_{i}
\end{aligned}
$$

where $x_{i} \in \mathbb{R}^{n}, u_{i} \in \mathbb{R}^{m}, y_{i} \in \mathbb{R}^{p}$ are respectively the state, input and output vectors, $E, A \in \mathbb{R}^{n \times n}, B \in$ $\mathbb{R}^{n \times m}, C \in \mathbb{R}^{p \times n}$, and

$$
\begin{gathered}
\Delta^{\alpha} x_{i}=\sum_{j=0}^{i}(-1)^{j}\left(\begin{array}{l}
\alpha \\
j
\end{array}\right) x_{i-j}, \\
\left(\begin{array}{l}
\alpha \\
j
\end{array}\right)= \begin{cases}1 & \text { for } j=0, \\
\frac{\alpha(\alpha-1) \ldots(\alpha-j+1)}{j !} & \text { for } j=1,2, \ldots\end{cases}
\end{gathered}
$$

is the fractional $\alpha$-order difference of $x_{i}$.

It is assumed that

$$
\operatorname{det}[E \lambda-A] \neq 0 \quad \text { for some } s \in \mathbb{C} \text {. }
$$

Definition 1. For any matrix $\bar{E}=[E \lambda-A]^{-1} E \in \mathbb{R}^{n \times n}$ there exists a unique Drazin inverse $\bar{E}^{D} \in \mathbb{R}^{n \times n}$ defined by the conditions

$$
\begin{aligned}
\bar{E}^{D} \bar{E} & =\bar{E} \bar{E}^{D}, \\
\bar{E}^{D} \bar{E} \bar{E}^{D} & =\bar{E}^{D}, \\
\bar{E}^{D} \bar{E}^{\mu+1} & =\bar{E}^{\mu},
\end{aligned}
$$

where $\mu$ is the smallest nonnegative integer such that

$$
\operatorname{rank} \bar{E}^{\mu}=\operatorname{rank} \bar{E}^{\mu+1},
$$

and $\lambda$ is chosen so that (2) is satisfied.

It is easy to check that for the matrices

$$
\begin{gathered}
P=\bar{E}^{D}, \quad \bar{E}=\bar{E}^{D}[E \lambda-A]^{-1} E, \\
\hat{A}=\bar{E}^{D}[E \lambda-A]^{-1} A
\end{gathered}
$$

the following relations hold:

$$
\begin{aligned}
& P^{k}=P \quad \text { for } \quad k=2,3, \ldots, \\
& P \hat{A}=\hat{A} P=\hat{A}
\end{aligned}
$$

Premultiplying (1a) by the matrix $\bar{E}^{D}[E \lambda-A]^{-1}$, we obtain

$$
P \Delta^{\alpha} x_{i+1}=\hat{A} x_{i}+\hat{B} u_{i}
$$

where

$$
\hat{B}=\bar{E}^{D}[E \lambda-A]^{-1} B
$$

Substituting (1c) into (6a), we obtain

$$
P x_{i+1}=\hat{A}_{\alpha} x_{i}+\sum_{j=2}^{i+1} c_{j} x_{i-j+1}+\hat{B} u_{i}, \quad i \in \mathbb{Z}_{+},
$$

where

$$
\begin{aligned}
\hat{A}_{\alpha} & =\hat{A}+P \alpha \\
c_{j} & =(-1)^{j+1}\left(\begin{array}{l}
\alpha \\
j
\end{array}\right), \quad j=1,2, \ldots .
\end{aligned}
$$

From (7a) it follows that the fractional system is equivalent to the descriptor system with an increasing number of delays.

Theorem 1. The solution of Eqn. (7a) has the form

$$
\begin{aligned}
& x_{i}=\Phi_{i} x_{0}+\sum_{j=0}^{i-1} \Phi_{i-j-1} \hat{B} u_{j}, \quad i \in \mathbb{Z}_{+}, \\
& x_{0}=\operatorname{Im} P=P d, \quad d \in \mathbb{R}^{n}: \text { arbitrary, }
\end{aligned}
$$

where the matrix $\Phi_{j}$ is given by

$$
\Phi_{j+1}=\hat{A}_{\alpha} \Phi_{j}+\sum_{k=2}^{j+1} c_{k} \Phi_{j-k+1}, \Phi_{0}=I_{n},
$$

and $\hat{A}_{\alpha}, c_{k}$ are defined by (7b) and (7c), respectively.

Proof. Using (7) and (8), it is easy to verify that

$$
\begin{aligned}
P x_{i+1}= & P\left[\Phi_{i+1} x_{0}+\sum_{j=0}^{i} \Phi_{i-j} \hat{B} u_{j}\right] \\
= & P \hat{A}_{\alpha} \Phi_{i} x_{0}+\sum_{j=0}^{i} P \Phi_{i-j} \hat{B} u_{j} \\
& +\sum_{k=2}^{i+1} c_{k} \Phi_{i-k+1} x_{0} \\
= & \hat{A}_{\alpha}\left[\Phi_{i} x_{0}+\sum_{j=0}^{i-1} \Phi_{i-j-1} \hat{B} u_{j}\right] \\
& +\sum_{j=2}^{i+1} c_{j} x_{i-j+1}+\hat{B} u_{i},
\end{aligned}
$$

since, by (5b), $P \hat{A}_{\alpha}=\hat{A}_{\alpha}$. Therefore, the solution (8) satisfies Eqn. (7a).

Example 1. Consider the fractional descriptor system (1a) with

$$
\begin{array}{ll}
E=\left[\begin{array}{cc}
0 & 0 \\
-1 & 1
\end{array}\right], \quad A=\left[\begin{array}{ll}
0 & 1 \\
0 & 0
\end{array}\right], \\
B=\left[\begin{array}{l}
0 \\
1
\end{array}\right], \quad u(t)=\left\{\begin{array}{lll}
1 & \text { for } & i \in \mathbb{Z}_{+}, \\
0 & \text { for } & i<0 .
\end{array}\right.
\end{array}
$$


The system satisfies the assumption (2) since

$$
\operatorname{det}[E \lambda-A]=\left|\begin{array}{cc}
0 & -1 \\
-\lambda & \lambda
\end{array}\right|=-\lambda .
$$

Choosing $\lambda=-1$ and using [10, we obtain

$$
\begin{aligned}
& \bar{E}=[E \lambda-A]^{-1} E=\left[\begin{array}{cc}
-1 & 1 \\
0 & 0
\end{array}\right], \\
& \bar{A}=[E \lambda-A]^{-1} A=\left[\begin{array}{cc}
0 & -1 \\
0 & -1
\end{array}\right] .
\end{aligned}
$$

In this case, the Drazin inverse matrix has the form

$$
\bar{E}^{D}=\bar{E}=\left[\begin{array}{cc}
-1 & 1 \\
0 & 0
\end{array}\right]
$$

and

$$
\begin{aligned}
\hat{A} & =\bar{E}^{D} \bar{A}=\left[\begin{array}{cc}
-1 & 1 \\
0 & 0
\end{array}\right]\left[\begin{array}{cc}
0 & -1 \\
0 & -1
\end{array}\right] \\
& =\left[\begin{array}{ll}
0 & 0 \\
0 & 0
\end{array}\right] \\
\hat{B} & =\bar{E}^{D}[E \lambda-A]^{-1} B \\
& =\left[\begin{array}{cc}
-1 & 1 \\
0 & 0
\end{array}\right]\left[\begin{array}{cc}
0 & -1 \\
1 & -1
\end{array}\right]^{-1}\left[\begin{array}{l}
0 \\
1
\end{array}\right]=\left[\begin{array}{c}
-1 \\
0
\end{array}\right] \\
\hat{A}_{\alpha} & =\hat{A}+P \alpha=\left[\begin{array}{cc}
\alpha & -\alpha \\
0 & 0
\end{array}\right] .
\end{aligned}
$$

Note that, in this case,

$$
P \hat{A}_{\alpha}=P^{2} \alpha=P \alpha=\left[\begin{array}{cc}
\alpha & -\alpha \\
0 & 0
\end{array}\right] .
$$

Using (8) and (14), we obtain

$$
\begin{aligned}
\Phi_{1}= & \hat{A}_{\alpha}=\left[\begin{array}{cc}
\alpha & -\alpha \\
0 & 0
\end{array}\right], \\
\Phi_{2}= & \hat{A}_{\alpha}^{2}+c_{2} I_{2}=\left[\begin{array}{cc}
\alpha & -\alpha \\
0 & 0
\end{array}\right]^{2} \\
& +\frac{\alpha(1-\alpha)}{2}\left[\begin{array}{cc}
1 & 0 \\
0 & 1
\end{array}\right], \\
\Phi_{3}= & \hat{A}_{\alpha}^{3}+c_{2} \hat{A}_{\alpha}+c_{3} I_{2}=\left[\begin{array}{cc}
\alpha & -\alpha \\
0 & 0
\end{array}\right]^{3} \\
& +\frac{\alpha(1-\alpha)}{2}\left[\begin{array}{cc}
\alpha & -\alpha \\
0 & 0
\end{array}\right] \\
& +\frac{\alpha(1-\alpha)(2-\alpha)}{6}\left[\begin{array}{ll}
1 & 0 \\
0 & 1
\end{array}\right],
\end{aligned}
$$

and

$$
\begin{aligned}
& x_{1}=\Phi_{1} x_{0}+\hat{B} u_{0}, \\
& x_{2}=\Phi_{2} x_{0}+\Phi_{1} \hat{B} u_{0}+\hat{B} u_{1}, \\
& x_{3}=\Phi_{3} x_{0}+\Phi_{2} \hat{B} u_{0}+\Phi_{1} \hat{B} u_{1}+\hat{B} u_{2},
\end{aligned}
$$

where

$$
x_{0}=\operatorname{Im} P=\operatorname{Im}\left[\begin{array}{cc}
1 & -1 \\
0 & 0
\end{array}\right]
$$

(the set of vectors $\left[\begin{array}{c}x_{10} \\ 0\end{array}\right]$, where $x_{10}$ is arbitrary).

Theorem 2. The solution $x_{i}$ of Eqn. (1a) satisfies

$$
P x_{i}=x_{i}, \quad i \in \mathbb{Z}_{+},
$$

that is, the solution $x_{i}$ starting from $x_{0}$ in the subspace $\operatorname{Im} P$ remains in this subspace for all $i \in \mathbb{Z}_{+}$.

Proof. From (8), we have

$$
\begin{aligned}
P x_{i} & =P \Phi_{i} x_{0}+\sum_{j=0}^{i-1} P \Phi_{i-j-1} \hat{B} u_{j} \\
& =\Phi_{i} x_{0}+\sum_{j=0}^{i-1} P \Phi_{i-j-1} \hat{B} u_{j}=x_{i},
\end{aligned}
$$

since $P \hat{A}_{\alpha}=\hat{A}_{\alpha}$ and

$$
\begin{aligned}
P \Phi_{i} & =P \hat{A}_{\alpha} \Phi_{i-1}+\sum_{k=1}^{i} c_{k} \Phi_{i-k} \\
& =\Phi_{i}, \quad i=1,2, \ldots
\end{aligned}
$$

Therefore, the solution $x_{i}, i \in \mathbb{Z}_{+}$, starting from $x_{0} \in$ $\operatorname{Im} P$, remains in this subspace for all $i \in \mathbb{Z}_{+}$.

Example 2. (Continuation of Example 1) In this case

$$
P=\left[\begin{array}{cc}
1 & -1 \\
0 & 0
\end{array}\right]
$$

and the subspace

$$
\operatorname{Im} P=P d=\left[\begin{array}{l}
d \\
0
\end{array}\right]
$$

consists of all vectors with a zero second component and (15) holds.

\section{Positivity of fractional descriptor linear discrete-time systems}

The following lemma will be used in our further discussion.

Lemma 1. For the fractional discrete-time linear system

$$
\Delta^{\alpha} z_{i+1}=M z_{i}, \quad M \in \mathbb{R}^{n \times n}, \quad 0<\alpha<1,
$$

the implication

$$
\begin{aligned}
F z_{0} \in \mathbb{R}_{+}^{p} \quad \text { then } \quad F z_{i} \in \mathbb{R}_{+}^{p} & \\
& \text { for } F \in \mathbb{R}^{p \times n}, \quad i \in \mathbb{Z}_{+}
\end{aligned}
$$

holds true if and only if there exists $H \in \mathbb{R}_{+}^{p \times p}$ such that 


$$
F M=H F \text {. }
$$

Proof. Premultiplying (21) by the matrix $F$, we obtain

$$
\Delta^{\alpha} F z_{i+1}=F M z_{i}, \quad i \in \mathbb{Z}_{+} .
$$

Equation (24) has the solution $F z_{i} \in \mathbb{R}_{+}^{p}, i \in \mathbb{Z}_{+}$if and only if (23) holds true. Note that the equation

$$
\Delta^{\alpha} F z_{i+1}=H F z_{i}, \quad i \in \mathbb{Z}_{+}
$$

has the solution $F z_{i} \in \mathbb{R}_{+}^{p}, i \in Z_{+}$if and only if $H \in$ $\mathbb{R}_{+}^{p \times p}$.

First, let us consider the autonomous fractional descriptor discrete-time system

$$
E \Delta^{\alpha} z_{i+1}=A z_{i}
$$

obtained from (1a) for $B u_{i}=0$.

Definition 2. The autonomous fractional descriptor system (26) is called (internally) positive if $x_{i} \in \mathbb{R}_{+}^{n}$, $i \in \mathbb{Z}_{+}$, for any admissible initial conditions $x_{0} \in \mathbb{R}_{+}^{n}$ $\left(x_{0} \in \operatorname{Im} P\right)$.

Theorem 3. The fractional descriptor system (26) is positive if and only if there exists a matrix $G \in \mathbb{R}^{n \times n}$ such that

$$
H=\hat{A}_{\alpha}+G\left(I_{n}-P\right) \in \mathbb{R}_{+}^{n \times n},
$$

where $\hat{A}_{\alpha}$ and $P$ are defined by (4).

Proof. By Lemma 1, the system (26) is positive if and only if there exists a matrix $H \in \mathbb{R}_{+}^{p \times p}$ such that

$$
\hat{A}_{\alpha}=H P \text {. }
$$

The solution of Eqn. (28) is given by (27) since, by (5b) and (5a), $\hat{A}_{\alpha} P=\hat{A}_{\alpha}, P^{2}=P$ and

$$
H P=\hat{A}_{\alpha} P+G\left(I_{n}-P\right) P=\hat{A}_{\alpha} P=\hat{A}_{\alpha} .
$$

This completes the proof.

Note that the system (26) can be positive even though the matrix $\hat{A}_{\alpha}$ is not nonnegative. If $\hat{A}_{\alpha} \in \mathbb{R}_{+}^{n \times n}$, then we have the following result.

Corollary 1. The fractional descriptor system 26) is positive if $\hat{A}_{\alpha} \in \mathbb{R}_{+}^{n \times n}$. In this case, we may choose in (27) $G=0$.

Example 3. (Continuation of Example 1) Consider the autonomous fractional system (26) with

$$
E=\left[\begin{array}{cc}
0 & 0 \\
-1 & 1
\end{array}\right], \quad A=\left[\begin{array}{ll}
0 & 1 \\
0 & 0
\end{array}\right], \quad 0<\alpha<1 .
$$

This system is positive since by Theorem 3 there exists a matrix $G \in \mathbb{R}^{2 \times 2}$ such that the condition (27) is satisfied. For (30) and

$$
G=\left[\begin{array}{ll}
0 & 1 \\
1 & 1
\end{array}\right]
$$

from (27) we obtain

$$
\begin{aligned}
H & =\hat{A}_{\alpha}+G\left(I_{n}-P\right) \\
& =\left[\begin{array}{ll}
0 & 1 \\
1 & 1
\end{array}\right]=\left[\begin{array}{cc}
\alpha & -\alpha \\
0 & 0
\end{array}\right]+\left[\begin{array}{ll}
0 & 1 \\
1 & 1
\end{array}\right]\left[\begin{array}{ll}
0 & 1 \\
0 & 1
\end{array}\right] \\
& =\left[\begin{array}{cc}
\alpha & 1-\alpha \\
0 & 2
\end{array}\right] \in \mathbb{R}_{+}^{2 \times 2}
\end{aligned}
$$

for any $\alpha \in(0,1)$. Note that the matrix $\hat{A}_{\alpha}$ has one negative entry.

In a general case, the positivity of the fractional descriptor system (1) is defined as follows.

Definition 3. The fractional descriptor system (1) is called (internally) positive if $x_{i} \in \mathbb{R}_{+}^{n}$ and $y_{i} \in \mathbb{R}_{+}^{p}$, $i \in \mathbb{Z}_{+}$, for any admissible initial conditions $x_{0} \in \mathbb{R}_{+}^{n}$ $\left(x_{0} \in \operatorname{Im} P\right)$ and all $u_{i} \in \mathbb{R}_{+}^{m}, i \in \mathbb{Z}_{+}$.

Theorem 4. The fractional descriptor system (1) is positive if and only if there exists a matrix $G \in \mathbb{R}^{n \times n}$ such that (27) holds true and

$$
\hat{B} \in \mathbb{R}_{+}^{n \times m}, \quad C \in \mathbb{R}_{+}^{p \times n} .
$$

Proof. The proof of (27) is the same as that of Theorem 3. Note that

$$
\sum_{j=0}^{i-1} \Phi_{i-j-1} \hat{B} u_{j} \in \mathbb{R}_{+}^{n} \quad \text { for } \quad i \in \mathbb{Z}_{+}
$$

if and only if $\hat{B} \in \mathbb{R}_{+}^{n \times m}$ since $u_{i} \in \mathbb{R}_{+}^{m}, i \in \mathbb{Z}_{+}$, is arbitrary. Similarly, $y_{i} \in \mathbb{R}_{+}^{p}, i \in \mathbb{Z}_{+}$if and only if $C \in$ $\mathbb{R}_{+}^{p \times n}$ since $x_{i} \in \mathbb{R}_{+}^{m}, i \in \mathbb{Z}_{+}$, can be arbitrary. This completes the proof.

Example 4. Consider the system (1) with

$$
\begin{array}{ll}
E=\left[\begin{array}{ll}
1 & 1 \\
0 & 0
\end{array}\right], \quad A=\left[\begin{array}{cc}
1 & 0.2 \\
0 & 1
\end{array}\right], \\
B=\left[\begin{array}{l}
1 \\
0
\end{array}\right], & C=\left[\begin{array}{ll}
1 & 0
\end{array}\right] .
\end{array}
$$

The assumption (2) is satisfied for $\lambda=0$ and

$$
\begin{aligned}
\bar{E} & =[-A]^{-1} E=\left[\begin{array}{cc}
-1 & -0.2 \\
0 & -1
\end{array}\right]^{-1}\left[\begin{array}{ll}
1 & 1 \\
0 & 0
\end{array}\right] \\
& =\left[\begin{array}{cc}
-1 & -1 \\
0 & 0
\end{array}\right], \\
\bar{A} & =[-A]^{-1} A=\left[\begin{array}{cc}
-1 & 0 \\
0 & -1
\end{array}\right],
\end{aligned}
$$


and

$$
\bar{E}^{D}=\bar{E}=\left[\begin{array}{cc}
-1 & -1 \\
0 & 0
\end{array}\right]
$$

Using (36) and (37), we obtain

$$
\begin{aligned}
P & =\bar{E}^{D} \bar{E}=\bar{E}=\left[\begin{array}{ll}
1 & 1 \\
0 & 0
\end{array}\right], \\
\hat{A} & =\bar{E}^{D} \bar{A}=\left[\begin{array}{ll}
1 & 1 \\
0 & 0
\end{array}\right], \\
\hat{A}_{\alpha} & =\hat{A}+P \alpha=\left[\begin{array}{cc}
\alpha+1 & \alpha+1 \\
0 & 0
\end{array}\right] .
\end{aligned}
$$

Note that the condition (27) for the positivity of the system is satisfied for $G=0$. Therefore, by Theorem 4 the system is positive since the matrices $\hat{B}$ and $C$ defined by (35) satisfy the condition (33).

\section{Concluding remarks}

The positivity of fractional descriptor linear discrete-time systems was investigated. The solution to the state equation of the fractional descriptor linear discrete-time system was derived (Theorems 1 and 2). Necessary and sufficient conditions for the positivity of fractional descriptor linear discrete-time systems was established (Theorems 3 and 4). The discussion were illustrated with numerical examples.

\section{Acknowledgment}

This work was supported by the National Science Centre in Poland under the grant no. 2017/27/B/ST7/02443.

\section{References}

Ali, R.M. and Napp, D. (2012). Characterization and stability of autonomous positive descriptor systems, IEEE Transactions on Automatic Control 57(10): 2668-2673.

Berman, A. and Plemmons, R.J. (1994). Nonnegative Matrices in the Mathematical Sciences, SIAM, New York, NY.

Borawski, K. (2018). Analysis of the positivity of descriptor continuous-time linear systems by the use of Drazin inverse matrix method, in R. Szewczyk et al. (Eds.), Automation 2018, Advances in Intelligent Systems and Computing, Vol. 743, Springer, Cham, pp. 172-182.

Busłowicz, M. (2008). Stability of linear continuous-time fractional order systems with delays of the retarded type, Bulletin of the Polish Academy of Sciences: Technical Sciences 56(4): 319-324.

Busłowicz, M. (2012). Stability analysis of continuous-time linear systems consisting of $n$ subsystems with different fractional orders, Bulletin of the Polish Academy of Sciences: Technical Sciences 60(2): 279-284.
Busłowicz, M. and Kaczorek, T. (2009). Simple conditions for practical stability of positive fractional discrete-time linear systems, International Journal of Applied Mathematics and Computer Science 19(2): 263-269, DOI: 10.2478/v10006-009-0022-6.

Farina, L. and Rinaldi, S. (2000). Positive Linear Systems: Theory and Applications, J. Wiley, New York, NY.

Kaczorek, T. (1993). Theory of Control and Systems, Polish Scientific Publishers, Warsaw, (in Polish).

Kaczorek, T. (1997). Positive singular discrete-time linear systems, Bulletin of the Polish Academy of Sciences: Technical Sciences 45(4): 619-631.

Kaczorek, T. (2002). Positive $1 D$ and $2 D$ Systems, Springer, London.

Kaczorek, T. (2008). Fractional positive continuous-time linear systems and their reachability, International Journal of Applied Mathematics and Computer Science 18(2): 223-228, DOI: 10.2478/v10006-008-0020-0.

Kaczorek, T. (2010). Positive linear systems with different fractional orders, Bulletin of the Polish Academy of Sciences: Technical Sciences 58(3): 453-458.

Kaczorek, T. (2011a). Positive linear systems consisting of $n$ subsystems with different fractional orders, IEEE Transactions on Circuits and Systems 58(7): 1203-1210.

Kaczorek, T. (2011b). Selected Problems of Fractional Systems Theory, Springer, Berlin.

Kaczorek, T. (2012). Positive fractional continuous-time linear systems with singular pencils, Bulletin of the Polish Academy of Sciences: Technical Sciences 60(1): 9-12.

Kaczorek, T. (2013). Application of the Drazin inverse to the analysis of descriptor fractional discrete-time linear systems with regular pencils, International Journal of Applied Mathematics and Computer Science 23(1): 29-33, DOI: 10.2478/amcs-2013-0003.

Kaczorek, T. (2014). Descriptor positive discrete-time and continuous-time nonlinear systems, Proceedings of SPIE 9290, DOI: $10.1117 / 12.2074558$.

Kaczorek, T. (2015a). Analysis of positivity and stability of discrete-time and continuous-time nonlinear systems, Computational Problems of Electrical Engineering 5(1): $11-16$.

Kaczorek, T. (2015b). Positivity and stability of discrete-time nonlinear systems, IEEE 2nd International Conference on Cybernetics, Gdynia, Poland, pp. 156-159.

Kaczorek, T. (2015c). Stability of fractional positive nonlinear systems, Archives of Control Sciences 25(4): 491-496, DOI: 10.1515/acsc-2015-0031.

Kaczorek, T. (2016a). Analysis of positivity and stability of fractional discrete-time nonlinear systems, Bulletin of the Polish Academy of Sciences: Technical Sciences 64(3): 491-494.

Kaczorek, T. (2016b). Drazin inverse matrix method for fractional descriptor discrete-time linear systems, Bulletin of the Polish Academy of Sciences: Technical Sciences 64(2): 395-399. 
Kaczorek, T. (2018). Stability of interval positive continuous-time linear systems, Bulletin of the Polish Academy of Sciences: Technical Sciences 66(1): 2018.

Kaczorek, T. (2019). Positivity and stability of standard and fractional descriptor continuous-time linear and nonlinear systems, International Journal of Nonlinear Sciences and Numerical Simulation 19(3-4): 299-307.

Klamka, J. (2002). Positive controllability of positive dynamical systems, Proceedings of the American Control Conference, ACC-2000, Anchorage, AK, USA, pp. 4395-4400.

Mitkowski, W. (2000). Remarks on stability of positive linear systems, Control and Cybernetics 29(1): 295-304.

Mitkowski, W. (2008). Dynamical properties of Metzler systems, Bulletin of the Polish Academy of Sciences, Technical Sciences 56(4): 309-312.

Sajewski, Ł. (2016a). Descriptor fractional discrete-time linear system and its solution-Comparison of three different methods, in R. Szewczyk et al. (Eds.), Challenges in Automation, Robotics and Measurement Techniques, Advances in Intelligent Systems and Computing, Vol. 440, Springer, Berlin/Heidelberg, pp. 37-50.

Sajewski, Ł. (2016). Descriptor fractional discrete-time linear system with two different fractional orders and its solution, Bulletin of the Polish Academy of Sciences, Technical Sciences 64(1): 15-20.

Zhang, H., Xie, D., Zhang, H. and Wang, G. (2014a). Stability analysis for discrete-time switched systems with unstable subsystems by a mode-dependent average dwell time approach, ISA Transactions 53(10): 1081-1086.

Zhang, J, Han, Z., Wu, H. and Hung, J. (2014b). Robust stabilization of discrete-time positive switched systems with uncertainties and average dwell time switching, Circuits Systems and Signal Processing 33(1): 71-95.
Xiang-Jun, W., Zheng-Mao, W. and Jun-Guo, L. (2008) Stability analysis of a class of nonlinear fractional-order systems, IEEE Transactions Circuits and Systems II: Express Briefs 55(11): 1178-1182.

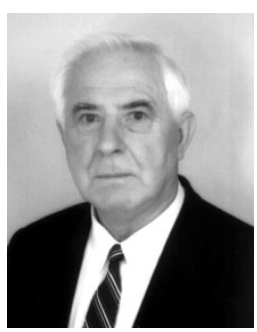

Tadeusz Kaczorek received his $\mathrm{MSc}, \mathrm{PhD}$ and DSc degrees in electrical engineering from the Warsaw University of Technology in 1956, 1962 and 1964, respectively. In 1971 he became a professor and in 1974 a full professor at the same university. Since 2003 he has been a professor at the Białystok University of Technology. In 1986 he was elected a corresponding member and in 1996 a full member of the Polish Academy of Sciences. In 2004 he was elected an honorary member of the Hungarian Academy of Sciences. He has been granted honorary doctorates by 13 universities. His research interests cover systems theory, especially singular multidimensional systems, positive multidimensional systems, singular positive 1D and 2D systems, as well as positive fractional $1 \mathrm{D}$ and $2 \mathrm{D}$ systems. He initiated research in the field of singular 2D, positive 2D and positive fractional linear systems. $\mathrm{He}$ has published 28 books ( 8 in English) and over 1100 scientific papers. $\mathrm{He}$ has also supervised $69 \mathrm{PhD}$ theses. He is the editor-in-chief of the Bulletin of the Polish Academy of Sciences: Technical Sciences and a member of editorial boards of ten international journals.

Received: 22 October 2018 Revised: 31 January 2019 Accepted: 5 March 2019 